\title{
Combination of the ginsenosides $R b 3$ and $R b 2$ exerts protective effects against myocardial ischemia reperfusion injury in rats
}

\author{
XIAOMIN LIU ${ }^{1,2}$, YICHUAN JIANG ${ }^{1}$, WENWEN FU ${ }^{1}$, XIAOFENG YU ${ }^{1}$ and DAYUN SUI ${ }^{1}$ \\ ${ }^{1}$ Department of Pharmacology, School of Pharmaceutical Sciences, Jilin University, Changchun, Jilin 130021; \\ ${ }^{2}$ Department of Pharmacy, Affiliated Hospital of Jilin Medical College, Jilin City, Jilin 132013, P.R. China
}

Received June 15, 2019; Accepted October 29, 2019

DOI: $10.3892 /$ ijmm.2019.4414

\begin{abstract}
Ginsenoside Rb3 (G-Rb3) has been demonstrated to alleviate myocardial ischemia reperfusion injury (MIRI); however, it is difficult to separate G-Rb2 from its isomer $\mathrm{G}-\mathrm{Rb} 3$. The current study aimed to compare the cardioprotective effects of G-Rb3 and the concomitant use of G-Rb3 and $\mathrm{G}-\mathrm{Rb} 2(\mathrm{G}-\mathrm{Rb} 3 / \mathrm{Rb} 2)$ on MIRI in rats. A rat model of MIRI was established by ligation of the left anterior descending coronary artery and the rats were randomly divided into five groups. Prior to MIRI, G-Rb3/Rb2 (20 mg/kg), G-Rb3 (20 mg/kg) and diltiazem (DLZ; $20 \mathrm{mg} / \mathrm{kg}$, as a positive control) were orally administered to the rats once a day for 3 consecutive days. After $30 \mathrm{~min}$ of ischemia and $120 \mathrm{~min}$ of reperfusion, cardiac function, infarct size, cardiac marker enzymes, antioxidative parameters, inflammatory factors, histopathological changes, cardiomyocyte apoptosis, and B-cell lymphoma 2 (Bcl-2), Bcl-2-associated $\mathrm{X}$ protein and caspase-3 expression were determined using a multi-channel physiological recording system, nitrotetrazolium blue chloride, biochemical kits, radioimmunoassay kits, hematoxylin and eosin, terminal deoxynucleotidyl-transferase-mediated dUTP nick end labeling assay, immunohistochemistry and reverse transcription-quantitative $\mathrm{PCR}$, respectively. The results indicated that treatment with $\mathrm{G}-\mathrm{Rb} 3 / \mathrm{Rb} 2$ significantly protected rats against MIRI, as shown by improved cardiac function, reduced myocardial ischemic area, decreased serum activities of aspartate aminotransferase, lactate dehydrogenase and creatine kinase $\mathrm{MB}$, decreased serum concentrations of interleukin- 6 and tumor necrosis factor- $\alpha$, decreased malondialdehyde concentration in myocardial tissues, increased activities of superoxide dismutase, glutathione peroxidase and catalase in myocardial tissues, reduced histopathological changes in myocardial tissues, reduced number of apoptotic
\end{abstract}

Correspondence to: Professor Dayun Sui, Department of Pharmacology, School of Pharmaceutical Sciences, Jilin University, 1266 Fujin Road, Changchun, Jilin 130021, P.R. China

E-mail: suidy@jlu.edu.cn

Key words: ginsenosides $\mathrm{Rb} 3$ and $\mathrm{Rb} 2$, myocardial ischemia reperfusion injury, oxidative stress, inflammation, apoptosis cardiomyocytes, and changes in the expression levels of caspase-3, Bcl-2 and Bax. In addition, the effects of treatment with G-Rb3/Rb2, G-Rb3 or DLZ were equivalent. The protective effects of G-Rb3/Rb2 on MIRI were similar to those of $\mathrm{G}-\mathrm{Rb} 3$ in terms of oxidative stress, inflammatory factors and inhibition of cardiomyocyte apoptosis. Therefore, G-Rb3/Rb2 may be developed as a concomitant treatment for MIRI.

\section{Introduction}

According to the World Health Organization, coronary artery disease is the most common cause of clinical mortality worldwide $(1,2)$. Acute myocardial infarction (AMI) mainly occurs upon coronary occlusion, which is caused by detachment and rupture of unstable atherosclerotic plaques $(3,4)$. At present, percutaneous coronary intervention (5), coronary artery bypass grafting $(6,7)$ or thrombolytic therapy (8) are effective treatments against AMI, which work by restoring blood perfusion and oxygen supply. However, coronary reperfusion can cause secondary damage to ischemic tissues, which is known as myocardial ischemia reperfusion injury (MIRI). MIRI usually leads to irreversible damage to ultrastructure, cell death and increased infarct size; therefore, MIRI has become a major obstacle in clinical treatment $(9,10)$.

It is widely accepted that the mechanism underlying MIRI is mainly associated with a sudden increase in the generation of reactive oxygen species (ROS), neutrophil infiltration, nitric oxide aggregation, inflammatory reaction, $\mathrm{Ca}^{2+}$ overload, endoplasmic reticulum stress, disordered energy metabolism, cardiomyocyte apoptosis, autophagy, necrosis and necroptosis induced by prolonged IRI $(11,12)$. Cardiomyocyte apoptosis, which is a type of programmed cell death, is a rare event in healthy myocardium; however, it is the earliest and predominant form of cell death in infarcted myocardium and has been associated with MIRI (13). Following MIRI, although the blood supply is restored, oxygen free radicals and overload of $\mathrm{Ca}^{2+}$ produced during ischemia initiate cardiomyocyte apoptosis. Previous studies have reported that the structural and functional damage to myocardial cells may primarily result from apoptosis during MIRI. Preventing the occurrence of cardiomyocyte apoptosis has become a target for certain drugs that interfere with MIRI $(14,15)$. It has been reported that curcumin is a potent cardioprotective compound, which may have beneficial effects on MIRI by reducing oxidative stress, 
preventing inflammation and inhibiting cardiomyocyte apoptosis (16). Numerous studies have indicated that traditional Chinese medicine (TCM) exerts a potent cardioprotective effect on MIRI and may be used as a therapeutic approach for its treatment $(17,18)$.

As a drug used in TCM, ginseng is a perennial herb belonging to the Araliaceae family and is a species within the genus Panax. Ginseng has been used in Asian and Western countries since ancient times. Among the 17 different species assigned to this genus, the major commercial ginseng varieties, namely Panax ginseng, Panax notoginseng and Panax quinquefolium L., are most commonly used as medicines (19). The primary constituents of ginseng are ginsenosides, triterpenoids and saponins, which have been reported to exert strong pharmacological activities, including anti-diabetic, anti-fatigue, anti-depressant and anti-cancer properties (20,21). Among the 200 types of ginsenosides and saponins, Rb1, Rb2, Rg1, $\mathrm{Rg} 3, \mathrm{Rd}, \mathrm{Re}, \mathrm{Rh} 1$ and Rh2 have been studied most extensively. Ginsenoside Rb2 (G-Rb2) and G-Rb3 share the same backbone structure of four-ring dammarane, but they differ in their carbohydrate moieties at $\mathrm{C} 20$; G-Rb2 possesses $\alpha$-L-arabinose (pyranose), whereas G-Rb3 possesses $\beta$-D-xylose (Fig. 1) (22).

It is difficult to separate G-Rb2 from its isomer G-Rb3. The process of G-Rb2 removal is complex, which may markedly increase development costs, making it unsuitable for large-scale production. Previous studies have demonstrated that G-Rb3 and G-Rb2 exert cardioprotective effects when used alone $(23,24)$. Therefore, the combined use of G-Rb3 and G-Rb2 (G-Rb3/Rb2) may simplify the isolation process, reduce production costs and even exert synergistic effects. A Chinese national patent (CN201210474548.0) has been applied for the method of extraction of G-Rb3/Rb2, and the content of G-Rb3 and G-Rb2 in the obtained extract was 95-80 and 5-20\%, respectively, as determined by high-performance liquid chromatography (HPLC) (25). The present study aimed to compare the protective effect of G-Rb3/Rb2 and G-Rb3 on MIRI in rats.

Diltiazem (DLZ) is a representative non-dihydropyridine calcium antagonist, which possesses mild peripheral vasodilatation properties, and can increase coronary and renal blood flow. DLZ has been widely used in the treatment of ischemic heart disease and hypertension $(26,27)$. Previous studies have reported that DLZ can significantly reduce myocardial cell injury, apoptosis induced by ischemia reperfusion and myocardial infarct size $(28,29)$. Therefore, DLZ was selected as a positive control in the present study.

\section{Materials and methods}

Chemical reagents. Nitrotetrazolium blue chloride (NBT; cat. no. N6876) was purchased from Sigma-Aldrich; Merck KGaA. Aspartate aminotransferase (AST; cat. no. 2401157), lactate dehydrogenase (LDH; cat. no. 2401131) and creatine kinase MB (CK-MB; cat. no. 20162400887) isoenzyme biochemical kits were obtained from Biosino Bio-technology \& Science, Inc. Malondialdehyde (MDA; cat. no. A003-1-2), superoxide dismutase (SOD; cat. no. A001-1-2), glutathione peroxidase (GSH-Px; cat. no. A005-1-2) and catalase (CAT; A007-2-1) biochemical assay kits were purchased from Nanjing Jiancheng Bioengineering Institute. Tumor necrosis factor- $\alpha$ (TNF- $\alpha$; cat. no. KYRD-0011) and interleukin-6 (IL-6; cat. no. KYRD-0017) radioimmunoassay kits were obtained from Beijing KangyuanRuide Biotech. Co., Ltd. Terminal deoxynucleotidyl-transferase-mediated dUTP nick end labeling (TUNEL) apoptosis detection kit (cat. no. 11684817910) was purchased from Sigma-Aldrich; Merck KGaA. Antibodies against B-cell lymphoma 2 (Bcl-2; cat. no. 15071), Bcl-2-associated X protein (Bax; cat. no. 14796) and caspase-3 (cat. no. 9661) were obtained from Cell Signaling Technology, Inc. for immunohistochemistry (IHC). PerfectStart ${ }^{\mathrm{TM}}$ Green qPCR SuperMix kit (cat. no. AQ601-01) was obtained from TransGen Biotech Co., Ltd. All other chemicals were of analytical grade.

Drug preparation. G-Rb3/Rb2 was extracted and identified by Professor Yanping Chen (Department of Natural Medicinal Chemistry, Jilin University, Changchun, China), as follows: Total ginsenosides were extracted from the caudexes or leaves of Panax quinquefolium L., as previously described (30), and were then dissolved in ethanol (1:10-50). Subsequently, an equal volume of $0.5 \% \mathrm{NaOH}$ ethanol solution was added to obtain panaxadiol saponins, which were subjected to silica gel column chromatography and eluted with chloroform:methanol:ethyl acetate: $\mathrm{H}_{2} \mathrm{O}$ (15:22:40:10, the upper layer). Upon elution, the eluent solvent was recovered and the sample was dried to a constant weight to obtain G-Rb3/Rb2. The content of G-Rb3 and G-Rb2 in the obtained extract was 95-80 and 5-20\%, respectively, as determined by HPLC.

G-Rb3 was prepared by Professor Yanping Chen as described previously (30). All specimens were retained at the herbarium of Jilin University. As the positive control, DLZ was purchased from Tianjin Tanabe Seiyaku Co., Ltd.

Animals. The present study was approved by the Ethics Committee of Jilin University and was conducted in accordance with the Guide for the Care and Use of Laboratory Animals published by the National Institutes of Health (31). A total of 84 healthy male Sprague-Dawley rats (weight, 220-240 g; age, 7-8 weeks) were obtained from the Experimental Animal Center of Jilin University. All rats were housed in an environment at a constant temperature of $22-24^{\circ} \mathrm{C}$ and relative humidity of $45-55 \%$ under a 12-h light/dark cycle with free access to food and water. Prior to the start of the experiment, the rats were acclimated to their environment for 1 week.

Experimental protocols. All 84 healthy rats were randomly divided into five groups: i) Sham surgery [sham surgery and treatment with double distilled (dd) $\mathrm{H}_{2} \mathrm{O}$; ; ii) MIRI (MIRI surgery and treatment with $\mathrm{ddH}_{2} \mathrm{O}$ ); iii) $\mathrm{G}-\mathrm{Rb} 3 / \mathrm{Rb} 2$ (20 mg/kg); iv) G-Rb3 (20 mg/kg); and v) DLZ (20 mg/kg) as a positive control. G-Rb3/Rb2, G-Rb3 and DLZ were dissolved in $\mathrm{dd}_{2} \mathrm{O}$ prior to administration. The drugs and $\mathrm{ddH}_{2} \mathrm{O}$ were orally administered to the rats once a day for 3 consecutive days.

A MIRI rat model was established by trained personnel as follows: Briefly, 30 min after the final dose of drug administration, rats were anesthetized with $3 \%$ isoflurane in a closed chamber. During surgery, anesthesia was maintained with isoflurane (2-2.5\% in oxygen) using a precision vaporizer. The parameters evaluated to ensure the animals were fully anes- 


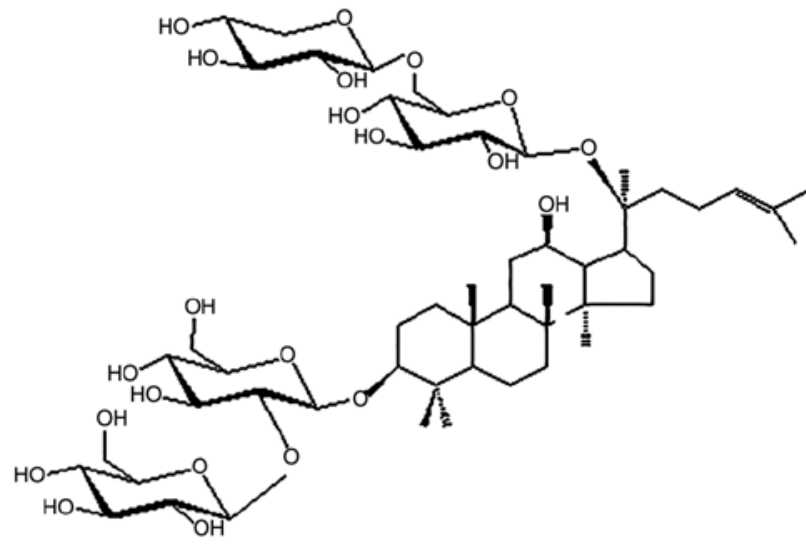

Ginsenoside Rb3

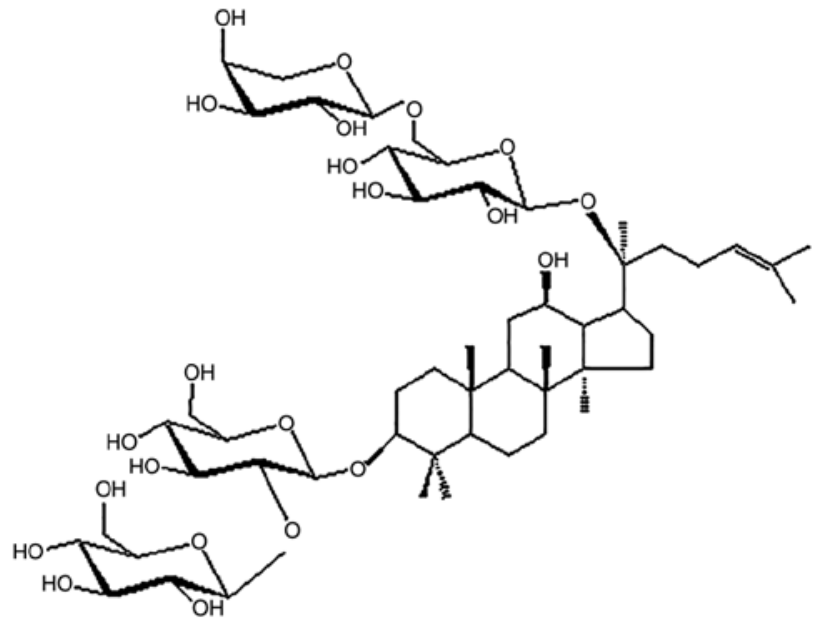

Ginsenoside Rb2

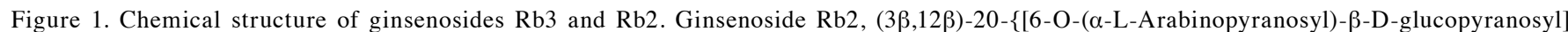

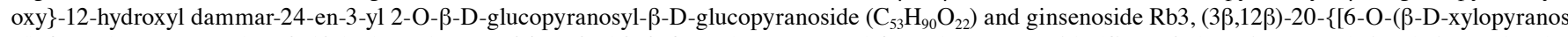
yl)- $\beta$-D-glucopyranosyl]oxy\}-12-hydroxydammar-24-en-3-yl 2-O- $\beta$-D-glucopyranosyl- $\beta$-D-glucopyranoside $\left(\mathrm{C}_{53} \mathrm{H}_{90} \mathrm{O}_{22}\right)$ are isomers; their relative molecular weights are 1,079.2685.

thetized prior to heart exposure were righting reflex and paw withdrawal reflex. Subsequently, a left parasternal incision was made between the third and fourth intercostal muscles to expose the rat heart, and the left anterior descending coronary artery was ligated with a 6-0 operative silk thread. After $30 \mathrm{~min}$ of ischemia, reperfusion was performed for $120 \mathrm{~min}$ by releasing the thread. The rats in the sham group underwent the same procedures, with the exception that the left anterior descending coronary artery was not ligated. During the $30 \mathrm{~min}$ of ischemia, ventricular fibrillation and ventricular tachycardia may occur in rats; therefore chest compressions were administered at the early stage (32). However, when this occurred more than three times and the rats could not be resuscitated by first aid, it was considered that the rats had reached humane endpoints and were euthanized using $\mathrm{CO}_{2}$ inhalation; the flow rate of $\mathrm{CO}_{2}$ displaced $25 \%$ of the chamber volume per minute.

Determination of cardiac function. After $120 \mathrm{~min}$ of reperfusion, the rats were anesthetized with an intraperitoneal injection of sodium pentobarbital $(40 \mathrm{mg} / \mathrm{kg}$; Sigma-Aldrich; Merck $\mathrm{KGaA}$ ); the bilateral common carotid arteries were isolated and cannulated into the left ventricle from the right common carotid artery using a 2-F polyethylene catheter. Subsequently, heart rate (HR), systolic blood pressure (SBP), diastolic blood pressure (DBP), left ventricular systolic pressure (LVSP), left ventricular end diastolic pressure (LVEDP), maximal rate of the increase of left ventricular pressure and maximal rate of the decrease of left ventricular pressure $( \pm \mathrm{dp} / \mathrm{dtmax})$ were measured using a multi-channel physiological recording system (RM-6000; Nihon Kohden Corporation).

Determination of AST, $C K-M B$ and LDHactivities in the serum. According to a previously described protocol (33), serum was prepared as follows: Following the determination of cardiac functional parameters, while rats were still under anesthesia, $5 \mathrm{ml}$ blood samples were collected from the abdominal aorta of each rat. The blood samples were completely coagulated by incubation at room temperature for $1 \mathrm{~h}$, and were then centrifuged at $1,000 \mathrm{x} \mathrm{g}$ for $10 \mathrm{~min}$ at $4^{\circ} \mathrm{C}$. The supernatant was collected for analysis. Commercial biochemical assay kits and an automatic biochemical analyzer (COBAS-FARA; Roche Diagnostics) were used to measure the activities of AST, LDH and CK-MB, according to the manufacturer's protocols.

Measurement of infarct size. After blood sample collection, the heart of each rat was removed, in order to harvest the left ventricular myocardium, which was weighed and cut into four horizontal slices from the apex to the base. Subsequently, the slices were incubated with $0.5 \mathrm{mg} / \mathrm{ml} \mathrm{NBT}$ at $37^{\circ} \mathrm{C}$ for $10 \mathrm{~min}$ to achieve complete staining, which was shown as a purple-black color in normal myocardial tissues and colorless in ischemic myocardial tissues. The ischemic myocardial zone was identified, separated from normal myocardial tissues and weighed. The myocardial infarct size was calculated as the weight of ischemic myocardium zone/the weight of left ventricular myocardium.

Measurement of MDA expression, and SOD, GSH-Px and CAT activities in the left ventricle. The homogenate of the left ventricle was prepared as follows: In total, $1 \mathrm{~g}$ left ventricle tissues were homogenized by adding nine volumes of ice-cold saline and then centrifuged for $15 \mathrm{~min}$ at $1,500 \mathrm{x} \mathrm{g}$ and $4^{\circ} \mathrm{C}$. The supernatant was collected to analyze MDA content, and SOD, GSH-Px and CAT activities using biochemical kits and a spectrophotometer [7202B; Unico (Shanghai) Instrument Co., Ltd.], according to the manufacturer's protocols.

Determination of TNF- $\alpha$ and IL-6 levels in the serum. In order to determine alterations in the levels of inflammatory factors in the serum, TNF- $\alpha$ and IL- 6 contents were measured by radioimmunoassay kits (Beijing KangyuanRuide Biotech. Co., Ltd.) and a gamma-scintillation counter (DFM-96; Beijing Zongcheng Electromechanical Technology Development Co., Ltd.), according to the manufacturers' protocols. 
Histopathological examination via histology, TUNEL assay and $I H C$. Myocardial tissue samples were fixed in a $4 \%$ paraformaldehyde solution for $24 \mathrm{~h}$ at room temperature, dehydrated in a graded series of ethanol $(50,75,85,95$ and $100 \%$ ), and then embedded in paraffin. According to the experimental protocol, specimens were cut into $4-\mu \mathrm{m}$ sections and were then stained with hematoxylin for $5 \mathrm{~min}$, followed by $1 \%$ hydrochloric acid alcohol differentiation for $3 \mathrm{sec}$ and eosin staining for $3 \mathrm{~min}$ at room temperature. According to the manufacturer's protocol, myocardial cell apoptosis was detected using the TUNEL apoptosis detection kit (Roche Diagnostics), prior to being examined by a pathologist blinded to the experimental design, under a light microscope (Nikon E100; Nikon Corporation) at x200 magnification. The five fields of view were automatically selected and the percentage of apoptosis-positive cells was calculated for each field of view. The mean was calculated to obtain the percentage of apoptotic cells and was expressed as apoptotic index. Apoptotic index $(\%)=($ apoptotic nuclei count/total nucleus count) $\mathrm{x} 100 \%$.

The protein expression of Bax, Bcl-2 and caspase- 3 in myocardial tissues was detected by IHC. The samples were prepared as aforementioned and sliced into $4-\mu \mathrm{m}$ sections. Following dehydration and paraffin embedding, the sections were blocked in $3 \%$ methanol- $\mathrm{H}_{2} \mathrm{O}_{2}$ for $10 \mathrm{~min}$ and incubated with primary antibodies (1:500) against Bcl-2, Bax and caspase- 3 for immunostaining at room temperature for $2 \mathrm{~h}$. After washing, polyclonal goat anti-mouse and goat anti-rabbit immunoglobulin $\mathrm{G}$ secondary antibody (1:1,500; cat. no. IB-0021 and IB-0061; Beijing Dingguo Changsheng Biotech Co., Ltd.) was added for $1 \mathrm{~h}$ at room temperature. Subsequently, sections were washed prior to development with avidin-biotin-streptavidin complex (OriGene Technologies, Inc.) at $30^{\circ} \mathrm{C}$ for $20 \mathrm{~min}$ and diaminobenzidine chromogen for $15 \mathrm{~min}$ at room temperature. The images of the stained sections were captured using the aforementioned light microscope at x200 magnification. Brown staining was recognized as positive expression. Five areas of each sample from all experimental groups were randomly captured.

For examination of myocardial ultrastructure, heart samples were initially fixed in a $2.5 \%$ phosphate-buffered glutaraldehyde solution at $4^{\circ} \mathrm{C}$ for $4 \mathrm{~h}$ and then further fixed in a $1 \%$ phosphate-buffered osmium tetroxide solution at $4^{\circ} \mathrm{C}$ for $2 \mathrm{~h}$. After dehydration with graded ethanol, the samples were embedded in resin and polymerized at $72^{\circ} \mathrm{C}$ for $48 \mathrm{~h}$. Ultrathin sections $(50-70 \mathrm{~nm})$ were then stained with $10 \%$ uranyl acetate for $30 \mathrm{~min}$ and $0.1 \%$ lead acetate for $15 \mathrm{~min}$ at room temperature. The myocardial ultrastructure was observed under a transmission electron microscope (JEM-1200 EX; JEOL, Ltd.) at $\mathrm{x} 7,500$ magnification.

Detection of caspase-3, Bcl-2 and Bax mRNA expression. Reverse transcription-quantitative polymerase chain reaction (RT-qPCR) was used to determine the mRNA expression levels of Bax, Bcl-2 and caspase-3. According to a previously described protocol (34), total RNA (1 $\mu \mathrm{g})$ was extracted from the left ventricular myocardial tissues using TRIzol ${ }^{\circledR}$ reagent (cat. no. 15596018; Invitrogen; Thermo Fisher Scientific, Inc.), and then subjected to RT using a riboSCRIPT ${ }^{\mathrm{TM}}$ Reverse
Transcription kit (Guangzhou RiboBio Co., Ltd.). The RT temperature protocol used was $42^{\circ} \mathrm{C}$ for $60 \mathrm{~min}$ and $72^{\circ} \mathrm{C}$ for $10 \mathrm{~min}$. RT-qPCR was performed using a TransStart Green qPCR Super Mix kit (Beijing Transgen Biotech Co., Ltd.) and a qPCR system (Mx3000P; Agilent Technologies, Inc.) with $\beta$-actin as the housekeeping gene. The thermocycling conditions were as follows: Initial denaturation at $94^{\circ} \mathrm{C}$ for $30 \mathrm{sec}$, followed by 40 cycles at $94^{\circ} \mathrm{C}$ for $5 \mathrm{sec}, 60^{\circ} \mathrm{C}$ for $30 \mathrm{sec}$ and $72^{\circ} \mathrm{C}$ for $30 \mathrm{sec}$, and a final extension step at $72^{\circ} \mathrm{C}$ for $5 \mathrm{~min}$. The results were analyzed using the $2^{-\Delta \Delta \mathrm{Cq}}$ method (35). The following primer sequences were used: Caspase-3, forward 5'-TCCTCGTTTCCGTGTTTG-3', reverse 5'-CAGGGCATC TCCACTTTG-3'; Bcl-2, forward 5'-TTGCTTGGCTGGTTC TAC-3', reverse 5'-TCTATGCCCTACCTATGAG-3'; Bax, forward 5'-TAGCCACAGTGTTGTAAGC-3', reverse 5'-TCT GATCCGTCTCAATAGTC-3'; and $\beta$-actin, forward 5'-TTG TGCCTTGATAGTTCGC-3' and reverse 5'-GGAGTCCTT CTGACCCATAC-3'.

Statistical analysis. All data are presented as the mean \pm standard deviation of at least three independent replicates. One-way analysis of variance followed by the Tukey-Kramer post hoc test was carried out using GraphPad Prism 5 software (GraphPad Software, Inc.). P $<0.05$ was considered to indicate a statistically significant difference.

\section{Results}

Effects of $G-R b 3 / R b 2$ and $G-R b 3$ on cardiac function parameters. After $30 \mathrm{~min}$ of ischemia and $120 \mathrm{~min}$ of reperfusion, cardiac function parameters were measured to determinate the extent of MIRI. As shown in Fig. 2, compared with the sham rats, HR, SBP, DBP, LVSP and \pm dp/dtmax were reduced, whereas LVEDP was significantly increased, in MIRI rats $(\mathrm{P}<0.05)$. In addition, HR, SBP, DBP, LVSP and $\pm \mathrm{dp} / \mathrm{dtmax}$ were increased $(\mathrm{P}<0.05)$ following the administration of G-Rb3/Rb2 (20 mg/kg), G-Rb3 (20 mg/kg) and DLZ $(20 \mathrm{mg} / \mathrm{kg})$, whereas LVEDP was significantly reduced $(\mathrm{P}<0.05)$ following the administration of $\mathrm{G}-\mathrm{Rb} 3 / \mathrm{Rb} 2$ $(20 \mathrm{mg} / \mathrm{kg})$ and DLZ $(20 \mathrm{mg} / \mathrm{kg})$.

Effects of $G-R b 3 / R b 2$ and $G-R b 3$ on infarct size, and AST, $L D H$ and $C K-M B$ activities. MIRI resulted in augmented myocardial infarct size, and increased AST, LDH and CK-MB activities ( $\mathrm{P}<0.05)$. G-Rb3/Rb2 (20 mg/kg), G-Rb3 (20 mg/kg) and DLZ $(20 \mathrm{mg} / \mathrm{kg})$ significantly inhibited the MIRI-induced increase in myocardial infarct size, and AST, LDH and CK-MB activities $(\mathrm{P}<0.05$; Fig. 3$)$. There was no statistically significant difference among the three treatment groups.

Effects of $G-R b 3 / R b 2$ and $G-R b 3$ on MDA expression, and SOD, GSH-Px and CAT activities. Compared with in the sham group, a higher level of oxidative stress was detected in MIRI rats, as manifested by significantly increased MDA expression, and reduced SOD, GSH-Px and CAT activities $(\mathrm{P}<0.05)$. The level of MDA was reduced, whereas the activities of SOD, GSH-Px and CAT were elevated, following treatment with G-Rb3/Rb2 (20 mg/kg), G-Rb3 (20 mg/kg) or DLZ $(20 \mathrm{mg} / \mathrm{kg})$ $(\mathrm{P}<0.05$; Fig. 4A-D). All three treatment groups exhibited similar results. 

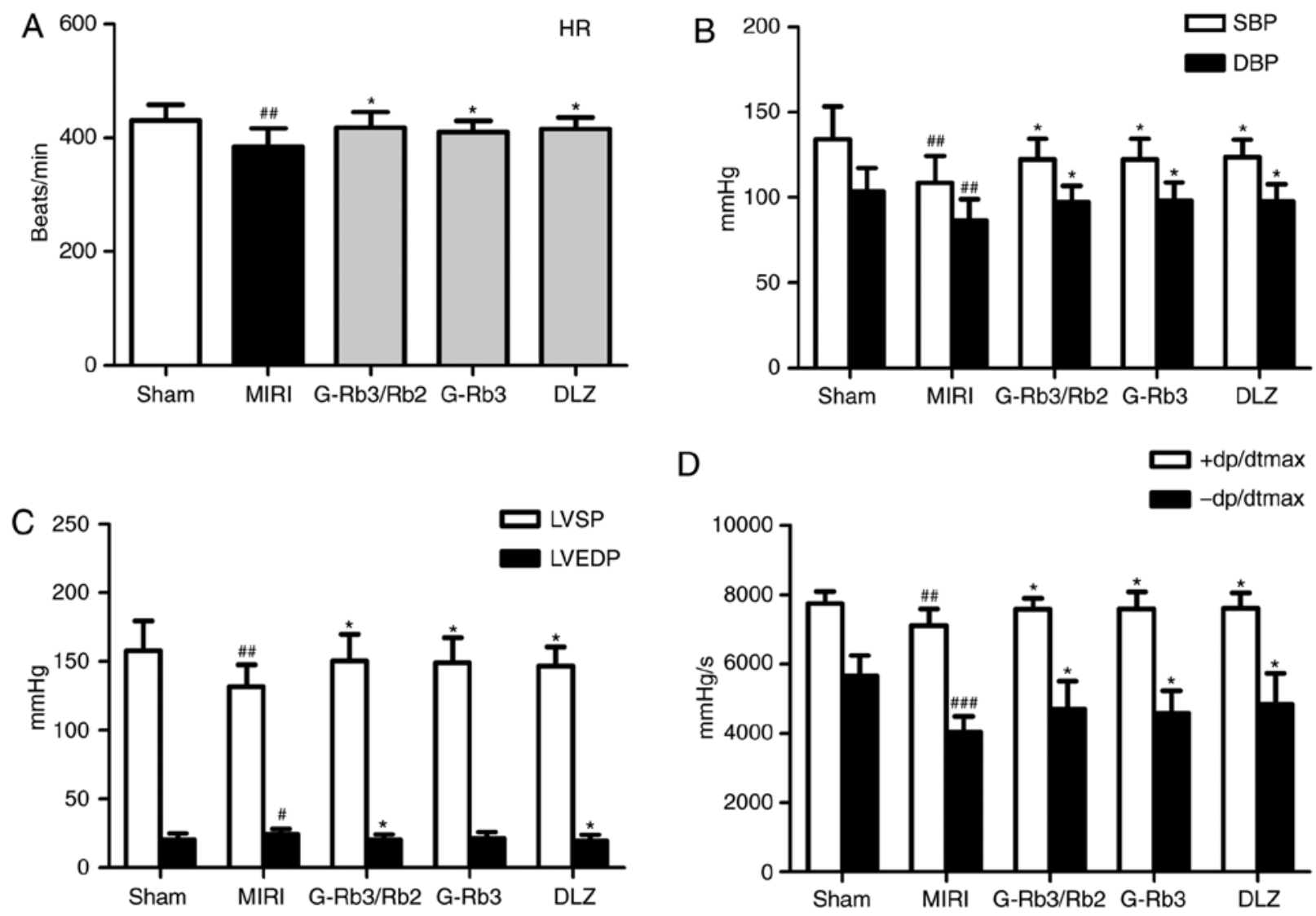

Figure 2. Effects of G-Rb3/Rb2 and G-Rb3 on cardiac function parameters in MIRI rats. (A) HR, (B) SBP and DBP, (C) LVSP and LVEDP, and (D) +dp/dtmax and -dp/dtmax were measured after $30 \mathrm{~min}$ of ischemia and $120 \mathrm{~min}$ of reperfusion $(\mathrm{n}=10 \mathrm{rats})$. Data are expressed as the mean \pm standard deviation. ${ }^{\#} \mathrm{P}<0.05$, ${ }^{\# \#} \mathrm{P}<0.01$ and ${ }^{\# \# \#} \mathrm{P}<0.001$ compared with the sham group; ${ }^{*} \mathrm{P}<0.05$ compared with the MIRI group. +dp/dtmax, maximal rate of the increase of left ventricular pressure; -dp/dtmax, maximal rate of the decrease of left ventricular pressure; DBP, diastolic blood pressure; DLZ, diltiazem; G, ginsenoside; HR, heart rate; LVEDP, left ventricular end diastolic pressure; LVSP, left ventricular systolic pressure; MIRI, myocardial ischemia and reperfusion injury; SBP, systolic blood pressure.

Effects of $G-R b 3 / R b 2$ and $G-R b 3$ on $T N F-\alpha$ and IL-6 levels. The serum levels of TNF- $\alpha$ and IL- 6 were determined using radioimmunoassay kits. The levels of TNF- $\alpha$ and IL- 6 in the MIRI group were significantly increased compared with in the sham group $(\mathrm{P}<0.05)$. Treatment with $\mathrm{G}-\mathrm{Rb} 3 / \mathrm{Rb} 2(20 \mathrm{mg} / \mathrm{kg})$, G-Rb3 $(20 \mathrm{mg} / \mathrm{kg})$ and DLZ $(20 \mathrm{mg} / \mathrm{kg})$ all attenuated the MIRI-induced increase in TNF- $\alpha$ and IL-6 levels $(\mathrm{P}<0.05$; Fig. 4E and F). The effects of the three treatment groups were similar.

Effects of $G-R b 3 / R b 2$ and $G-R b 3$ on histology, ultrastructural alterations, apoptosis and expression. No focal separation of myocardial fibers was detected in the myocardium of sham rats and these rats exhibited an intact myocardial cell membrane. However, widespread myocardial structure disorder and myocyte necrosis were observed in the myocardial tissue samples of the MIRI group. The other three groups exhibited mild necrosis and neutrophil granulocyte infiltration. These morphological alterations were not obviously alleviated compared with the MIRI group. The condition of myocardial injury was similar in these three groups (Fig. 5A).

According to the myocardial micrographs obtained by transmission electron microscopy (Fig. 5B), the myocardial fibers in the sham group were arranged in a regular manner, with a clear and ordered mitochondrial structure. The mito- chondria in the MIRI group were swollen with distorted cristae, vacuolar degeneration and large areas of cytoplasmic vacuolization caused by irregularity of myofilaments. The pathological alterations were alleviated following treatment with G-Rb3/Rb2 (20 mg/kg), G-Rb3 (20 mg/kg) or DLZ (20 $\mathrm{mg} / \mathrm{kg}$ ); however, these groups still exhibited slightly damaged cristae.

As shown in Fig. 5C, a TUNEL assay was used to detect apoptotic cells in myocardial tissues. The results revealed that the extent of DNA damage was significantly increased in the MIRI group but was significantly reduced in the G-Rb3/Rb2 (20 mg/kg), G-Rb3 (20 mg/kg) and DLZ (20 mg/kg) groups $(\mathrm{P}<0.05)$.

In addition, the IHC results indicated that Bcl-2 expression was significantly reduced, whereas Bax and caspase- 3 expression were increased, in the MIRI group. Administration of $\mathrm{G}-\mathrm{Rb} 3 / \mathrm{Rb} 2(20 \mathrm{mg} / \mathrm{kg}), \mathrm{G}-\mathrm{Rb} 3(20 \mathrm{mg} / \mathrm{kg})$ and DLZ $(20 \mathrm{mg} / \mathrm{kg}$ ) increased Bcl-2 expression, and reduced Bax and caspase-3 expression ( $\mathrm{P}<0.05$; Fig. 6A).

Effects of $G-R b 3 / R b 2$ and $G-R b 3$ on the mRNA expression levels of Bax, Bcl-2 and caspase-3. RT-qPCR was used to detect the effects of $\mathrm{G}-\mathrm{Rb} 3 / \mathrm{Rb} 2$ on the mRNA expression levels of Bax, Bcl-2 and caspase-3. Compared with in the sham group, rats in the MIRI group exhibited a significant reduction 
A
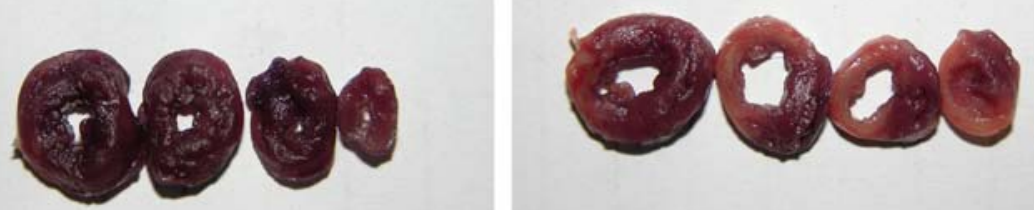

Sham

MIRI
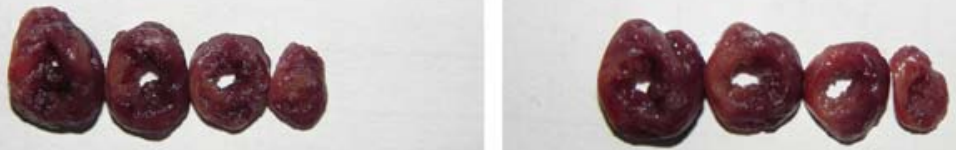

G-Rb3/Rb2

\section{G-Rb3}

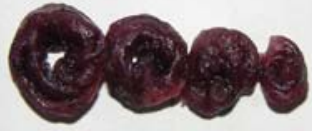

DLZ
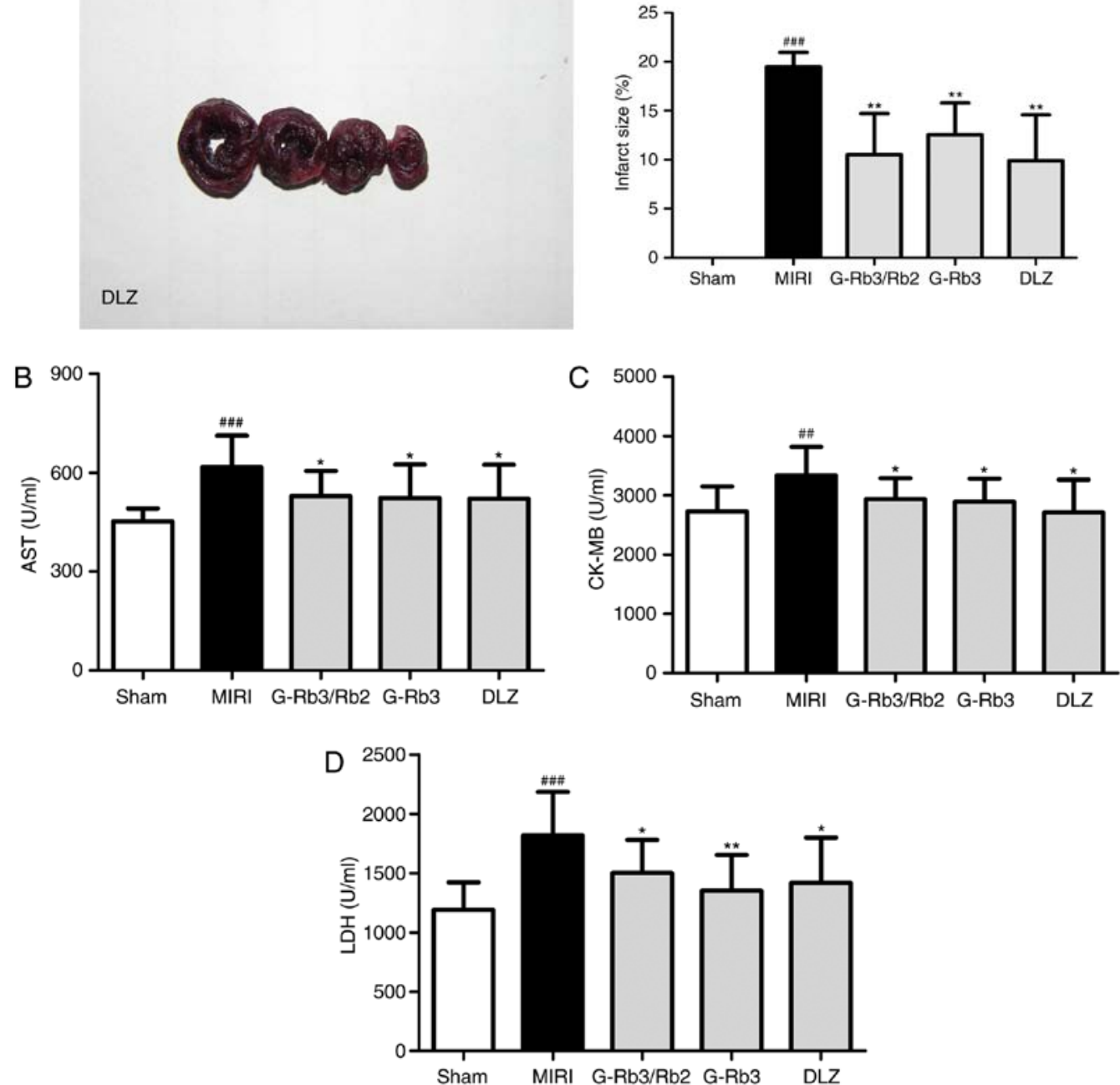

Figure 3. Effects of G-Rb3/Rb2 and G-Rb3 on myocardial infarct size, and the activities of AST, LDH and CK-MB in MIRI rats. (A) Representative myocardial cross-sections of nitrotetrazolium blue chloride-stained heart tissues from each group. Colorless sections indicate infarcted myocardium, whereas normal myocardium is stained purple-black ( $\mathrm{n}=4$ rats). (B) AST, (C) CK-MB and (D) LDH activities in the serum ( $\mathrm{n}=10$ rats). Data are expressed as the mean \pm standard deviation. ${ }^{\# \#} \mathrm{P}<0.01$ and ${ }^{\# \# \#} \mathrm{P}<0.001$, compared with the sham group; ${ }^{*} \mathrm{P}<0.05$ and ${ }^{* *} \mathrm{P}<0.01$, compared with the MIRI group. AST, aspartate aminotransferase; CK-MB, creatine kinase MB; DLZ, diltiazem; G, ginsenoside; LDH, lactate dehydrogenase; MIRI, myocardial ischemia and reperfusion injury. 

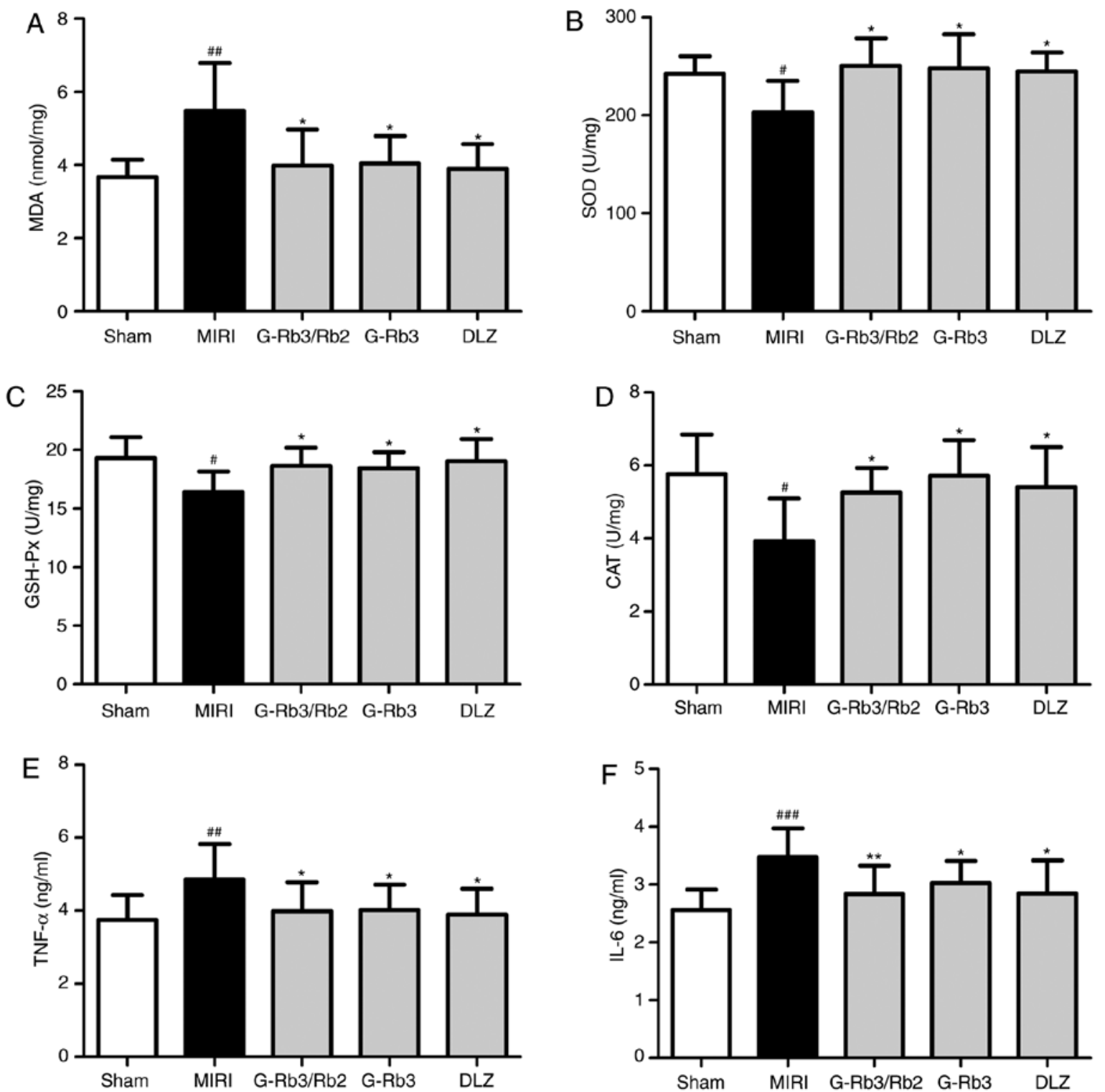

Figure 4. Effects of G-Rb3/Rb2 and G-Rb3 on oxidative stress and inflammatory factors. Levels of (A) MDA, and the activities of (B) SOD, (C) GSH-Px and (D) CAT in the left ventricular tissues were analyzed using biochemical assay kits (n=6 rats). Levels of (E) TNF- $\alpha$ and (F) IL-6 in the serum were detected by radioimmunoassay kits $\left(\mathrm{n}=10\right.$ rats). Data are expressed as the mean \pm standard deviation. ${ }^{*} \mathrm{P}<0.05,{ }^{\# \#} \mathrm{P}<0.01$ and ${ }^{\# \# \#} \mathrm{P}<0.001$ compared with the sham group; ${ }^{*} \mathrm{P}<0.05$ and ${ }^{* *} \mathrm{P}<0.01$ compared with the MIRI group. CAT, catalase; DLZ, diltiazem; G, ginsenoside; GSH-Px, glutathione peroxidase; IL-5, interleukin-6; MDA, malondialdehyde; MIRI, myocardial ischemia and reperfusion injury; SOD, superoxide dismutase; TNF- $\alpha$, tumor necrosis factor- $\alpha$.

in Bcl-2 mRNA expression, whereas Bax and caspase-3 mRNA expression was increased, as was the ratio of Bax/Bcl-2 expression $(\mathrm{P}<0.05)$. As shown in Fig. 6B-E, the mRNA expression levels of Bcl-2 were elevated, whereas the mRNA expression levels of Bax and caspase-3 were significantly reduced, along with a reduced ratio of $\mathrm{Bax} / \mathrm{Bcl}-2$ expression, following treatment with G-Rb3/Rb2 (20 mg/kg), G-Rb3 (20 mg/kg) and DLZ $(20 \mathrm{mg} / \mathrm{kg})(\mathrm{P}<0.05)$. No significant difference was observed among the three treatment groups.

\section{Discussion}

A rat model of MIRI was established by 30 min of ligation of the left anterior descending coronary artery followed by 120 min of reperfusion; this method has been widely used to research the underlying mechanisms of cardiomyocyte apop- tosis (36). Several studies have reported that rats are prone to death after ligation of the left anterior descending coronary artery due to arrhythmia; the mortality rate of this model can be as high as $60 \%(32,37)$. In the present study, 27 rats died and the mortality rate was $\sim 30 \%$; five rats died during the operation due to excess blood loss, 18 rats were euthanized due to reaching humane endpoints and four rats were deeply anesthetized and died of respiratory depression. It has been reported that the safety range of sodium pentobarbital is too narrow, which causes poor control of anesthesia depth, and some rats may die of respiratory depression even at the recommended anesthetic dose $(38,39)$. This rat model of MIRI was used to evaluate the cardioprotective effect of G-Rb3/Rb2 compared with that of G-Rb3. The most important indicators of cardiac function are hemodynamic parameters, such as HR, SBP, DBP, LVSP, LVEDP and $\pm \mathrm{dp} / \mathrm{dtmax}$. It was previously 
A
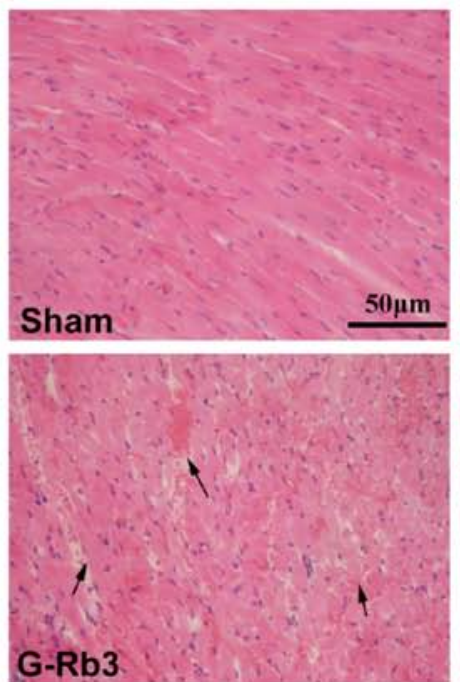

B
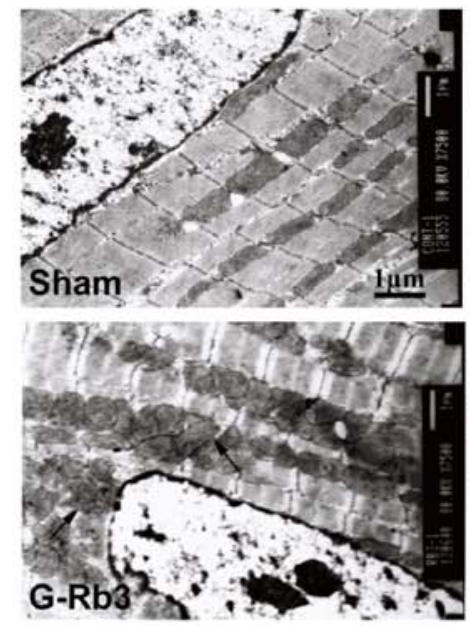

C
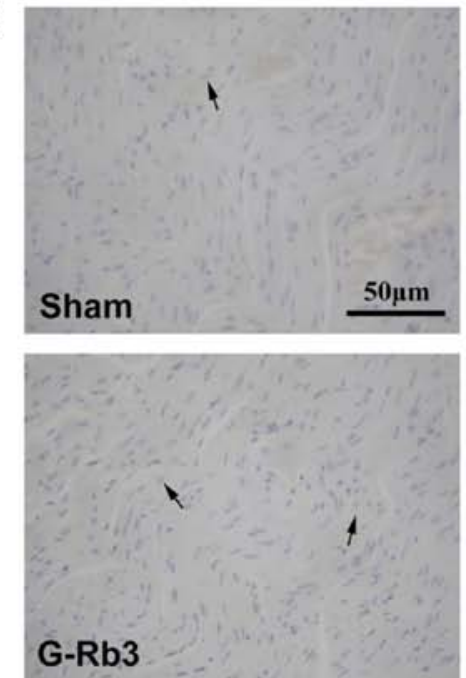
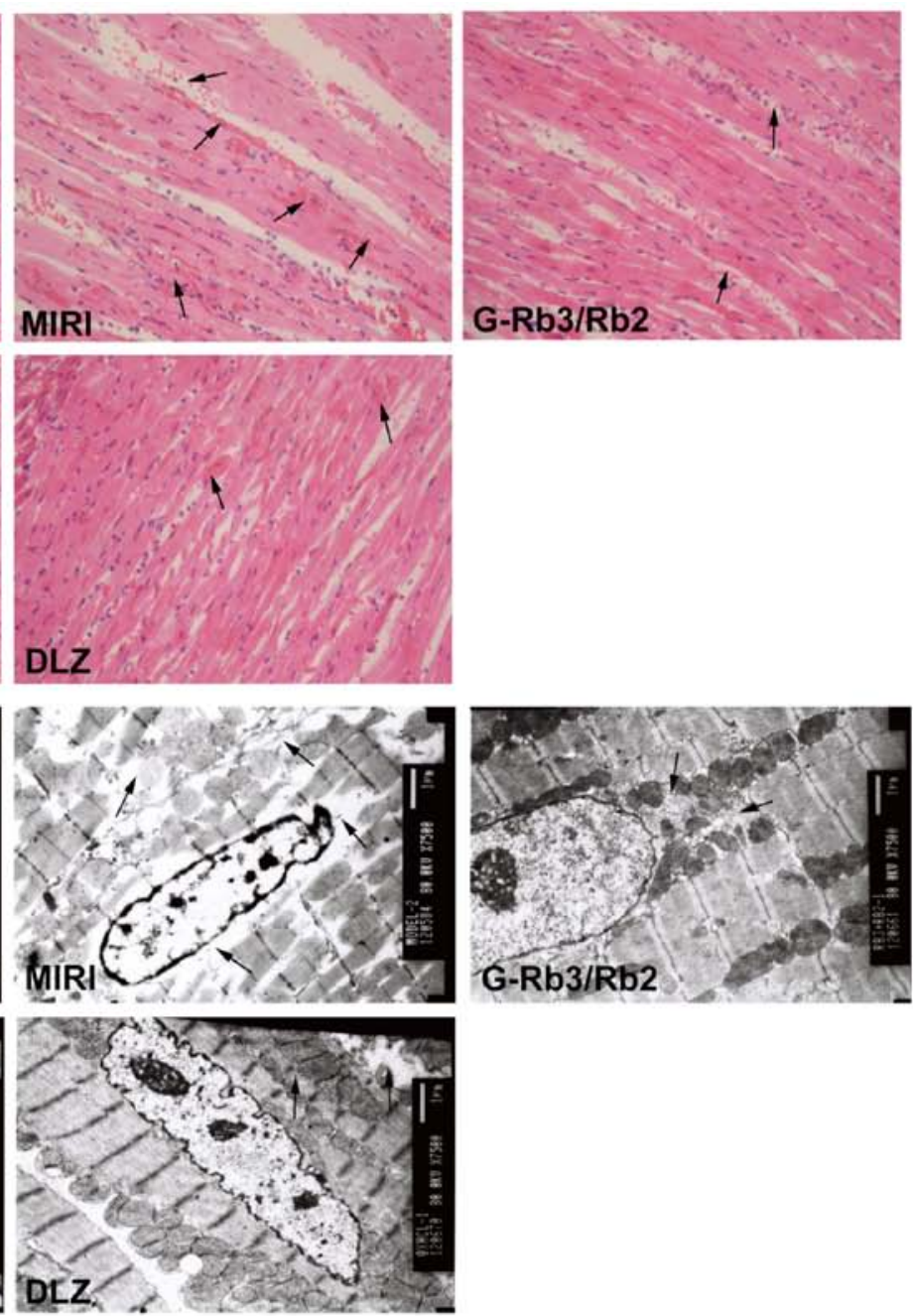
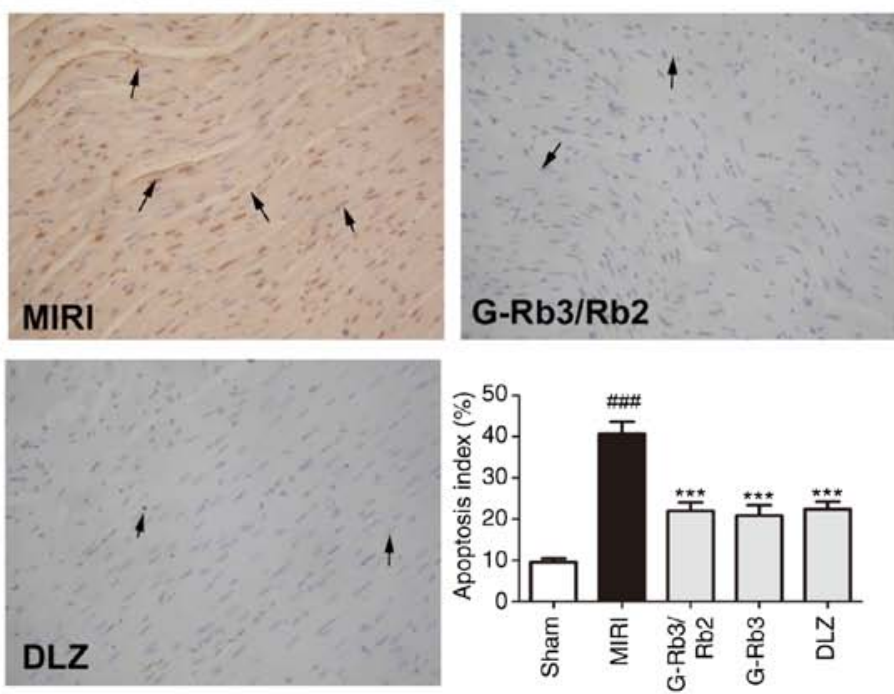

Figure 5. Effects of G-Rb3/Rb2 and G-Rb3 on the results of histological analysis and TUNEL assay. (A) Representative hematoxylin and eosin staining of myocardial tissues. The sections were examined under a light microscope at $\times 200$ magnification ( $\mathrm{n}=4$ rats). (B) Myocardial ultrastructure was observed under a transmission electron microscope at $\times 7,500$ magnification $(n=4$ rats). (C) TUNEL staining of myocardial tissues was examined under a light microscope at $\mathrm{x} 200$ magnification $(\mathrm{n}=4$ rats). Results of TUNEL assay were expressed as apoptosis index $(\%)$. All data are presented as the mean \pm standard deviation.

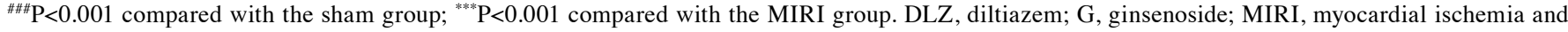
reperfusion injury; TUNEL, terminal deoxynucleotidyl-transferase-mediated dUTP nick end labeling.

reported that MIRI can lead to hemodynamic disorders and inhibition of heart function characterized by reduced HR, increased LVEDP, and decreased HR, SBP, DBP, LVSP and $\pm \mathrm{dp} / \mathrm{dtmax}(40)$. In the present study, hemodynamic parameters were incorporated into the experimental design to evaluate the effects of $\mathrm{G}-\mathrm{Rb} 3 / \mathrm{Rb} 2$ on cardiac function. Consistent with 
A

Bax

$\mathrm{Bcl}-2$

Caspase-3
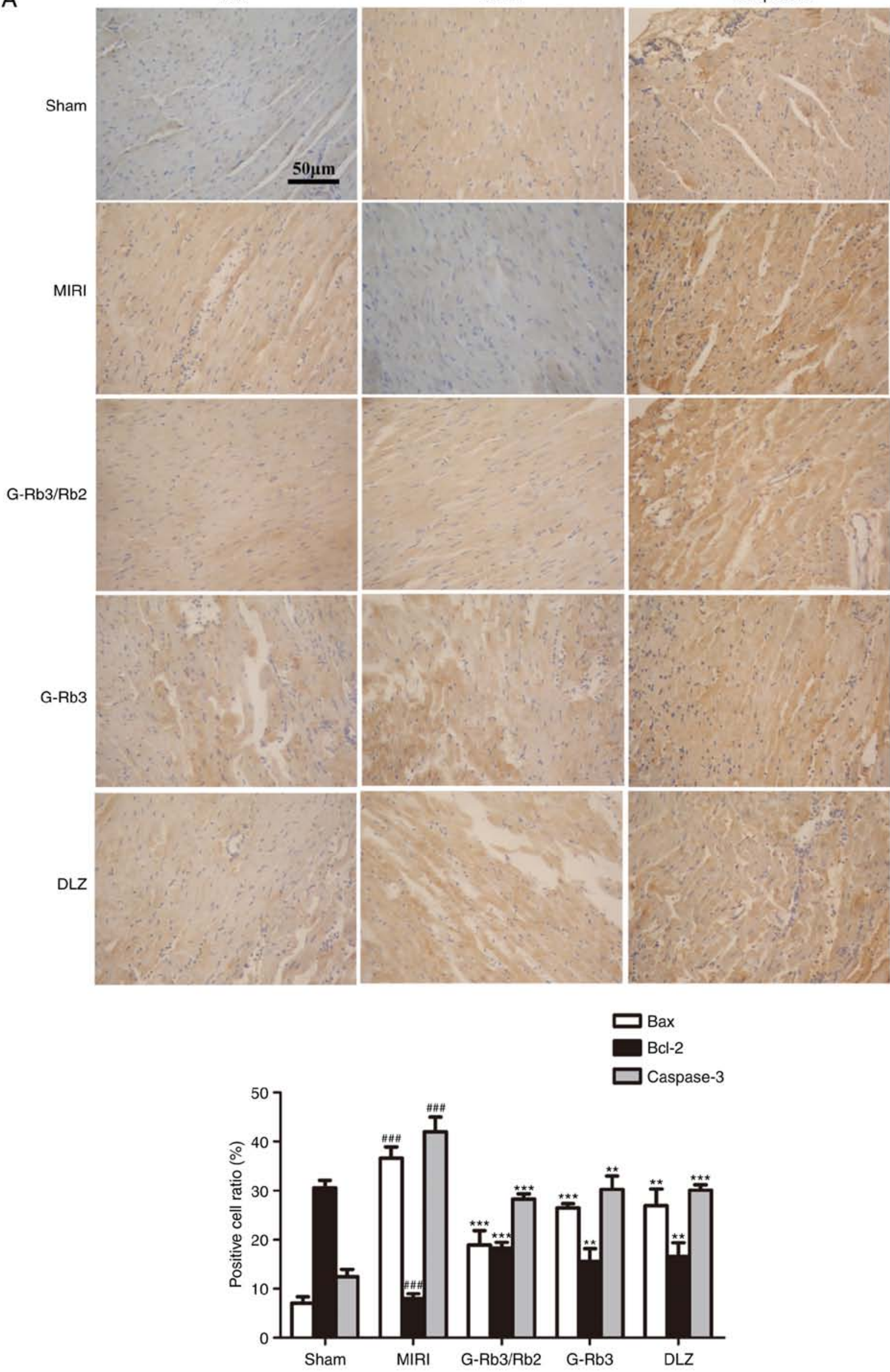

Figure 6. Effects of G-Rb3/Rb2 and G-Rb3 on caspase-3, Bcl-2 and Bax expression. (A) Representative IHC photomicrographs of myocardial tissues examined under a light microscope at $\mathrm{x} 200$ magnification ( $\mathrm{n}=4$ rats). The results were expressed as positive cell ratio (\%). 

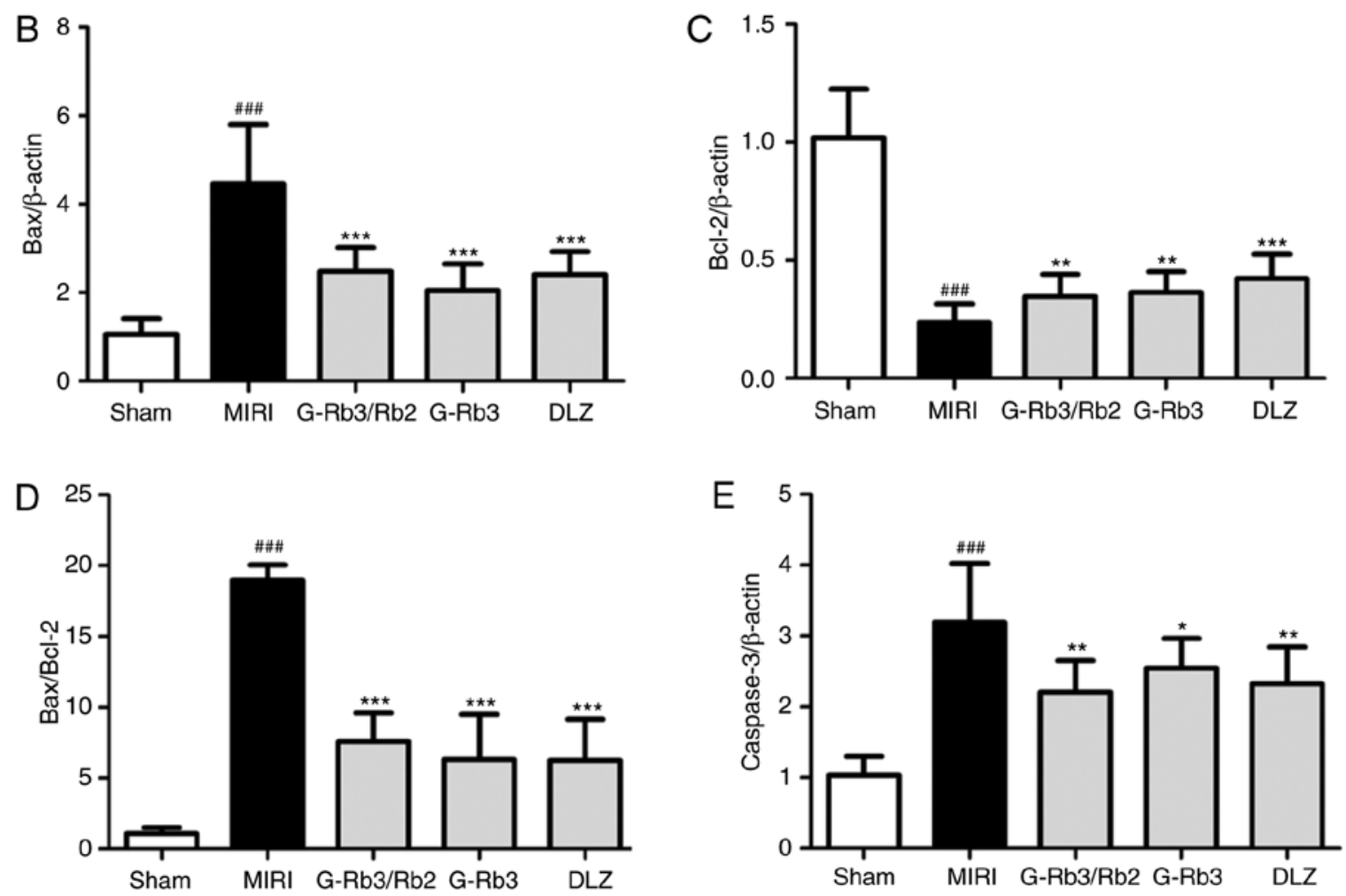

Figure 6. Continued. Effects of G-Rb3/Rb2 and G-Rb3 on caspase-3, Bcl-2 and Bax expression. (B-E) mRNA expression levels of (B) Bax, (C) Bcl-2 and (E) caspases-3 were detected by reverse transcription-quantitative polymerase chain reaction. (D) Ratio of Bax/Bcl-2 was calculated to assess the degree of myocardial tissue apoptosis ( $\mathrm{n}=4$ rats). Data are expressed as the mean \pm standard deviation. ${ }^{\# \# \# ~} \mathrm{P}<0.001$ compared with the sham group; ${ }^{*} \mathrm{P}<0.05$, ${ }^{* *} \mathrm{P}<0.01$ and ${ }^{* * * *} \mathrm{P}<0.001$, compared with the MIRI group. Bax, Bcl-2-associated X protein; Bcl-2, B-cell lymphoma 2; DLZ, diltiazem; G, ginsenoside; IHC, immunohistochemistry; MIRI, myocardial ischemia and reperfusion injury.

the results from previous studies, the MIRI rats exhibited significant cardiac dysfunction, whereas the administration of G-Rb3/Rb2 (20 mg/kg) and DLZ (20 mg/kg) significantly blocked these adverse changes. These results indicated that the concomitant use of G-Rb3 and G-Rb2 may ameliorate MIRI-induced impairment of cardiac function. No significant difference was observed between the two groups.

Infarct size is an important parameter for evaluating left ventricular function and the effectiveness of cardiovascular drugs. It can also be used to assess the condition and prognosis of coronary artery diseases. G-Rb3/Rb2 (20 mg/kg), G-Rb3 $(20 \mathrm{mg} / \mathrm{kg})$ and DLZ $(20 \mathrm{mg} / \mathrm{kg})$ significantly inhibited the MIRI-induced increase in myocardial infarct size. The integrity of the lipid membrane is important for maintaining cell structure and functionality, and increased levels of oxygen free radicals can lead to disordered cellular structures (41). As myocardial marker enzymes, AST, LDH and CK-MB can be released into the bloodstream when the cell membrane is ruptured in MIRI; therefore, changes in membrane integrity and the degree of myocardial injury can be reflected by the serum activities of AST, LDH and CK-MB (42). The results of the present study revealed a significant elevation in the activities of AST, LDH and CK-MB in MIRI rats. However, treatment with G-Rb3/Rb2 (20 mg/kg), G-Rb3 (20 mg/kg) and DLZ (20 mg/kg) significantly inhibited the MIRI-induced increase in AST, LDH and CK-MB activities. Taken together, these results demonstrated that the protective effects of these three drugs against MIRI may be mediated by increasing cardiomyocyte membrane stability, while decreasing the release of enzymes and myocardial infarct size. In addition, the three treatment groups exhibited similar effects.

Oxygen free radicals are a key factor in MIRI. A sudden increase in potent free radicals can be evoked within the first few minutes of reestablished blood flow when the onset of reperfusion reintroduces abundant oxygen. The release of free radicals in the early phase of reperfusion, in combination with the ischemia and reperfusion-induced decrease in antioxidant activity, renders the myocardium vulnerable to damage. During MIRI, an excessive amount of ROS is produced in the blood or in myocardial tissues to induce cardiomyocyte apoptosis $(43,44)$. Oxygen free radicals can also damage the myocardial cell membrane by peroxidizing the polyunsaturated fatty acids located in the cell membrane. As an end product of peroxidization of polyunsaturated fatty acids in the membrane, MDA is attacked by oxygen free radicals; therefore, the content of MDA can reflect the degree of lipid peroxidization. As free radical-scavenging enzymes, SOD, GSH-Px and CAT act as the first line of defense against oxidative stress by eliminating reactive oxygen radicals (45). In the present study, treatment with G-Rb3/Rb2 $(20 \mathrm{mg} / \mathrm{kg})$, G-Rb3 $(20 \mathrm{mg} / \mathrm{kg})$ and DLZ $(20 \mathrm{mg} / \mathrm{kg})$ reduced the MIRI-induced elevation in MDA content, and increased the activities of SOD, GSH-Px and CAT. These findings indicated that G-Rb3/Rb2, G-Rb3 and DLZ may protect against oxidative damage by enhancing the activity of endogenous antioxidant enzymes. Excessive inflammatory responses, including leukocyte exudation, edema, tissue necrosis and increased release of inflammatory cytokines (IL-6 and TNF- $\alpha$ ), are hallmarks of MIRI, which 
can activate the cytokine cascade, and promote the production of oxygen free radicals to activate neutrophils and induce the apoptosis of cardiomyocytes. IL-6 exerts a multifaceted effect on immune response, acute phase response, hematopoiesis and the nervous system (46). It has been reported that ischemia and reperfusion can significantly induce the production of IL-6 (47). TNF- $\alpha$ can inhibit myocardial contractile function, stimulate endothelial cells and neutrophils to produce adhesion molecules, and accelerate the adhesion between cells and intravascular coagulation, thus leading to necrosis and apoptosis of cardiomyocytes, which aggravate the degree of MIRI (48). Therefore, detection of TNF- $\alpha$ and IL- 6 concentration around the infarct area can indirectly reflect the degree of myocardial inflammatory infiltration and myocardial damage. According to the present data, G-Rb3/Rb2, G-Rb3 and DLZ treatment reduced the levels of IL- 6 and TNF- $\alpha$ in the blood, thus suggesting that the cardioprotective effects of these drugs may be mediated through their anti-inflammatory properties, and at least in part through the regulation of cardiomyocyte apoptosis via TNF- $\alpha$-mediated activation of the death receptor pathway.

In the present study, the myocardium underwent morphological alterations after $30 \mathrm{~min}$ of ischemia and $120 \mathrm{~min}$ of reperfusion. $H \& E$ staining revealed swollen myocardial cells with unclear boundaries, an enlarged intercellular space and irregular granularity in the cytoplasm, degenerated and swollen myocardial fibers, and marked infiltration of red blood cells and neutrophils. These morphological alterations were not obviously alleviated by G-Rb3/Rb2, G-Rb3 and DLZ treatment, which may be attributed to the duration of myocardial ischemia and reperfusion used in the present study and/or the experimental conditions of H\&E staining. In addition, serious damage was detected in the nuclei and mitochondria of cardiomyocytes under transmission electron microscopy, which is considered a 'gold standard' for determining apoptosis. G-Rb3/Rb2, G-Rb3 and DLZ treatment markedly alleviated myocardial ultrastructural damage; however, no marked alterations were observed under light microscopy after H\&E staining. These findings indicated that apoptosis occurred in the early stage of MIRI (after 120 min of reperfusion), consistent with previous studies $(49,50)$. It has been reported that MIRI and apoptosis appear in reperfused myocardium within $\leq 4 \mathrm{~h}$ (51). Serious damage to tissues was also observed by $H \& E$ staining and transmission electron microscopy after $30 \mathrm{~min}$ of ischemia and $120 \mathrm{~min}$ of reperfusion.

The TUNEL assay is capable of staining intact and single apoptotic nuclei or apoptotic body in situ, in order to accurately reflect the biochemical characteristics of apoptosis. The results of TUNEL staining indicated that G-Rb3/Rb2, G-Rb3 and DLZ could ameliorate apoptosis during ischemia reperfusion to reduce myocardial damage. A major pathological variant of MIRI is apoptosis. The Bcl-2 gene family regulates apoptosis via the mitochondrial pathway. Bax promotes apoptosis by promoting cytochrome $c$ release, activating caspases and forming a dimer with Bcl-2 to inhibit Bcl-2 activity (52). The anti-apoptotic effect of Bcl-2 is caused by its ability to inhibit activation of mitochondrial cytochrome $c$ and caspase-3; notably, the ratio of $\mathrm{Bax} / \mathrm{Bcl}-2$ can further reflect the regulation of apoptosis mediated by the Bcl-2 gene family (53). In the present study, the protein expression levels of caspase-3,
Bcl-2 and Bax were detected in myocardial tissues by IHC, which can directly and accurately locate the protein of interest in cells or tissues with high sensitivity in a qualitative manner. The IHC results indicated that $\mathrm{Bcl}-2$ expression was significantly reduced, whereas Bax and caspase-3 expression were increased, in the MIRI group. Administration of G-Rb3/Rb2, G-Rb3 and DLZ could increase Bcl-2 expression, and reduce Bax and caspase- 3 expression. In addition, the mRNA expression levels of Bax, Bcl-2 and caspase-3 were detected by RT-qPCR. The mRNA expression levels of Bax, Bcl-2 and caspase- 3 were similar to the protein expression levels detected by IHC. The ratio of Bax/Bcl-2 expression was increased after MIRI, and reduced following treatment with G-Rb3/Rb2, G-Rb3 and DLZ. The results indicated that G-Rb3/Rb2, G-Rb3 and DLZ may exert myocardial protective effects by affecting genes responsible for regulating apoptosis.

In conclusion, the protective effects of $\mathrm{G}-\mathrm{Rb} 3 / \mathrm{Rb} 2$ on MIRI were similar to those of G-Rb3. The underlying mechanisms may be attributed to regulation of oxidative stress and inflammatory factors, as well as inhibition of cardiomyocyte apoptosis, at least in part. Therefore, G-Rb3/Rb2 may be used as a concomitant treatment for MIRI.

\section{Acknowledgements}

The authors would like to thank Professor Yanping Chen (Department of Natural Medicinal Chemistry, Jilin University, Changchun, China) for providing G-Rb3/Rb2 and G-Rb3.

\section{Funding}

The present study received financial support from the National Natural Science Foundation of China (grant no. 81473378) and the Natural Science Foundation of Jilin Province (grant no. 20170101002JC).

\section{Availability of data and materials}

The datasets used and/or analyzed during the current study are available from the corresponding author on reasonable request.

\section{Authors' contributions}

DS and XL conceived and designed the study. YJ, WF and XY performed the experiments. XL wrote the paper. YJ and WF analyzed data, and reviewed and edited the manuscript. All authors read and approved the final manuscript.

\section{Ethics approval and consent to participate}

The present study was approved by the Ethics Committee of Jilin University.

\section{Patient consent for publication}

Not applicable.

\section{Competing interests}

The authors declare that they have no competing interests. 


\section{References}

1. Karimi M, Zare H, Bakhshian Nik A, Yazdani N, Hamrang M, Mohamed E, Sahandi Zangabad P, Moosavi Basri SM, Bakhtiari L and Hamblin MR: Nanotechnology in diagnosis and treatment of coronary artery disease. Nanomedicine (Lond) 11 513-530, 2016.

2. Krokhaleva Y and Vaseghi M: Update on prevention and treatment of sudden cardiac arrest. Trends Cardiovasc Med 29: 394-400, 2019

3. Zhou H, Ma Q, Zhu P, Ren J, Reiter RJ and Chen Y: Protective role of melatonin in cardiac ischemia-reperfusion injury: From pathogenesis to targeted therapy. J Pineal Res 64, 2018.

4. Rude RE, Muller JE and Braunwald E: Efforts to limit the size of myocardial infarcts. Ann Intern Med 95: 736-761, 1981.

5. Hausenloy DJ and Yellon DM: Myocardial ischemia-reperfusion injury: A neglected therapeutic target. J Clin Invest 123: 92-100, 2013 .

6. Nesher N, Zisman E, Wolf T, Sharony R, Bolotin G, David M, Uretzky G and Pizov R: Strict thermoregulation attenuates myocardial injury during coronary artery bypass graft surgery as reflected by reduced levels of cardiac-specific troponin I. Anesth Analg 96: 328-335, table of contents, 2003.

7. Jo MS, Lee J, Kim SY, Kwon HJ, Lee HK, Park DJ and Kim Y: Comparison between creatine kinase MB, heart-type fatty acid-binding protein, and cardiac troponin $\mathrm{T}$ for detecting myocardial ischemic injury after cardiac surgery. Clin Chim Acta 488: 174-178, 2019.

8. Virmani R, Forman MB and Kolodgie FD: Myocardial reperfusion injury. Histopathological effects of perfluorochemical. Circulation 81 (3 Suppl): IV57-IV68, 1990.

9. Hou H, Wang Y, Li Q, Li Z, Teng Y, Li J, Wang X, Chen J and Huang N: The role of RIP3 in cardiomyocyte necrosis induced by mitochondrial damage of myocardial ischemia-reperfusion. Acta Biochim Biophys Sin (Shanghai) 50: 1131-1140, 2018.

10. Ibáñez B, Heusch G, Ovize M and Van de Werf F: Evolving therapies for myocardial ischemia/reperfusion injury. $\mathrm{J}$ Am Coll Cardiol 65: 1454-1471, 2015.

11. Yu L, Zhang W, Huang C, Liang Q, Bao H, Gong Z, Xu M, Wang Z, Wen $\mathrm{M}$ and Cheng X: FoxO4 promotes myocardial ischemia-reperfusion injury: The role of oxidative stress-induced apoptosis. Am J Transl Res 10: 2890-2900, 2018.

12. Davidson SM, Ferdinandy P, Andreadou I, B $\varnothing$ tker HE, Heusch G Ibáñez B, Ovize M, Schulz R, Yellon DM, Hausenloy DJ, et al: Multitarget strategies to reduce myocardial ischemia/reperfusion injury: JACC review topic of the week. J Am Coll Cardiol 73: 89-99.2019.

13. Gottlieb RA, Burleson KO, Kloner RA, Babior BM and Engler RL: Reperfusion injury induces apoptosis in rabbit cardiomyocytes. J Clin Invest 94: 1621-1628, 1994.

14. Liu S, Ai Q, Feng K, Li Y and Liu X: The cardioprotective effect of dihydromyricetin prevents ischemia-reperfusion-induced apoptosis in vivo and in vitro via the PI3K/Akt and HIF-1 $\alpha$ signaling pathways. Apoptosis 21: 1366-1385, 2016.

15. Shu Z, Yang Y, Yang L, Jiang H, Yu X and Wang Y: Cardioprotective effects of dihydroquercetin against ischemia reperfusion injury by inhibiting oxidative stress and endoplasmic reticulum stress-induced apoptosis via the PI3K/Akt pathway. Food Funct 10: 203-215, 2019.

16. Mokhtari-Zaer A, Marefati N, Atkin SL, Butler AE and Sahebkar A: The protective role of curcumin in myocardial ischemia-reperfusion injury. J Cell Physiol 234: 214-222, 2018.

17. Sun J, Yu X, Huangpu $\mathrm{H}$ and Yao F: Ginsenoside Rb3 protects cardiomyocytes against hypoxia/reoxygenation injury via activating the antioxidation signaling pathway of PERK/Nrf2/HMOX1. Biomed Pharmacother 109: 254-261, 2019.

18. Liu K, Chen H, You QS, Ye Q, Wang F, Wang S, Zhang SL, Yu KJ and $\mathrm{Lu} \mathrm{Q}$ : Curcumin attenuates myocardial ischemia-reperfusion injury. Oncotarget 8: 112051-112059, 2017.

19. Wang CZ, Wu JA, McEntee E and Yuan CS: Saponins composition in American ginseng leaf and berry assayed by high-performance liquid chromatography. J Agric Food Chem 54: 2261-2266, 2006.

20. Kim JH: Pharmacological and medical applications of Panax ginseng and ginsenosides: A review for use in cardiovascular diseases. J Ginseng Res 42: 264-269, 2018.

21. Bai L, Gao J, Wei F, Zhao J, Wang D and Wei J: Therapeutic potential of ginsenosides as an adjuvant treatment for diabetes. Front Pharmacol 9: 423, 2018.
22. Wan JB, Yang FQ, Li SP, Wang YT and Cui XM: Chemical characteristics for different parts of Panax notoginseng using pressurized liquid extraction and HPLC-ELSD. J Pharm Biomed Anal 41: 1596-1601, 2006.

23. Liu X, Jiang Y, Yu X, Fu W, Zhang H and Sui D: Ginsenoside-Rb3 protects the myocardium from ischemia-reperfusion injury via the inhibition of apoptosis in rats. Exp Ther Med 8: 1751-1756, 2014.

24. Jiang Y, Zhong GG, Chen L and Ma XY: Influences of ginsenosides $\mathrm{Rb} 1, \mathrm{Rb} 2$, and $\mathrm{Rb} 3$ on electric and contractile activities of normal and damaged cultured myocardiocytes. Zhongguo Yao Li Xue Bao 13: 403-406, 1992.

25. Sui DY, Chen YP, Yu XF, Wang ZC, Qu SC and Ma XY: Application of composition containing ginsenoside-Rb3 and ginsenoside- $\mathrm{Rb} 2$ to treatment of heart and cerebral vascular diseases. CN201210474548.0, filed November 21, 2012.

26. Moukarbel GV, Ayoub CM and Abchee AB: Pharmacological therapy for myocardial reperfusion injury. Curr Opin Pharmacol 4: 147-153, 2004.

27. Pizzetti G, Mailhac A, Li Volsi L, Di Marco F, Lu C, Margonato A and Chierchia SL: Beneficial effects of diltiazem during myocardial reperfusion: A randomized trial in acute myocardial infarction. Ital Heart J 2: 757-765, 2001.

28. Herzog WR, Vogel RA, Schlossberg ML, Edenbaum LR, Scott HJ and Serebruany VL: Short-term low dose intracoronary diltiazem administered at the onset of reperfusion reduces myocardial infarct size. Int J Cardiol 59: 21-27, 1997.

29. Chen C, Chen W, Nong Z, Ma Y, Qiu S and Wu G: Cardioprotective effects of combined therapy with hyperbaric oxygen and diltiazem pretreatment on myocardial ischemia-reperfusion injury in rats. Cell Physiol Biochem 38: 2015-2029, 2016.

30. Shi Y,Han B, Yu X, Qu S and Sui D: Ginsenoside Rb3 ameliorates myocardial ischemia-reperfusion injury in rats. Pharm Biol 49: 900-906, 2011.

31. National Research Council (US) Committee for the Update of the Guide for the Care and Use of Laboratory Animals: Guide for the Care and Use of Laboratory Animals, 8th edition. National Academies Press, Washington, DC, 2011.

32. Cao Y, Li YK, Chen MQ and Yao KW: Research progress on preparation of rat models of acute myocardial infarction. Chin J Compat Med 27: 96-100, 2017.

33. Zhang H, Xu H, Xie H, Li F, Yu X and Sui D: Cardiovascular protective effects of IL-1 ra-Fc-IL-18BP on experimental myocardial infarction by inhibiting oxidative stress and inflammation in a rat model. Pharmazie 69: 769-774, 2014.

34. Dai S, Hong Y, Xu J, Lin Y, Si Q and Gu X: Ginsenoside Rb2 promotes glucose metabolism and attenuates fat accumulation via AKT-dependent mechanisms. Biomed Pharmacother 100: 93-100, 2018.

35. Livak KJ and Schmittgen TD: Analysis of relative gene expression data using real-time quantitative PCR and the 2(-Delta Delta C(T)) method. Methods 25: 402-408, 2001.

36. Maulik N, Yoshida T, Engelman RM, Deaton D, Flack JE III, Rousou JA and Das DK: Ischemic preconditioning attenuates apoptotic cell death associated with ischemia/reperfusion. Mol Cell Biochem 186: 139-145, 1998.

37. In: Animal Models of Human Diseases. EQ L (ed). People's Medical Publishing House (China), p113, 2008

38. Zeller A, Arras M, Jurd R and Rudolph U: Identification of a molecular target mediating the general anesthetic actions of pentobarbital. Mol Pharmacol 71: 852-859, 2007.

39. Archer DP, Samanani N and Roth SH: Small-dose pentobarbital enhances synaptic transmission in rat hippocampus. Anesth Analg 93: 1521-1525, table of contents, 2001.

40. Nassiri AA, Hakemi MS, Asadzadeh R, Faizei AM, Alatab S, Miri R and Yaseri M: Differences in cardiovascular disease risk factors associated with maximum and mean carotid intima-media thickness among hemodialysis patients. Iran J Kidney Dis 6: 203-208, 2012

41. Peng S, Wang Y, Zhou Y, Ma T, Wang Y, Li J, Huang F, Kou J, Qi L, Liu B and Liu K: Rare ginsenosides ameliorate lipid overload-induced myocardial insulin resistance via modulating metabolic flexibility. Phytomedicine 58: 152745, 2019.

42. Luo Y, Pan YZ, Zeng C, Li GL, Lei XM, Liu Z and Zhou SF: Altered serum creatine kinase level and cardiac function in ischemia-reperfusion injury during percutaneous coronary intervention. Med Sci Monit 17: CR474-CR479, 2011.

43. Ryter SW, Kim HP, Hoetzel A, Park JW, Nakahira K, Wang X and Choi AM: Mechanisms of cell death in oxidative stress. Antioxid Redox Signal 9: 49-89, 2007. 
44. Han SY, Li HX, Ma X, Zhang K, Ma ZZ and Tu PF: Protective effects of purified safflower extract on myocardial ischemia in vivo and in vitro. Phytomedicine 16: 694-702, 2009.

45. Zhai KF, Duan H, Khan GJ, Xu H, Han FK, Cao WG, Gao GZ, Shan LL and WeiZJ: Salicin from Alangium Chinense ameliorates rheumatoid arthritis by modulating the Nrf2-HO-1-ROS pathways. J Agric Food Chem 66: 6073-6082, 2018.

46. Lyu M, Cui Y, Zhao T, Ning Z, Ren J, Jin X, Fan G and Zhu Y: Tnfrsf $12 \mathrm{a}-\mathrm{mediated}$ atherosclerosis signaling and inflammatory response as a common protection mechanism of shuxuening injection against both myocardial and cerebral ischemia-reperfusion injuries. Front Pharmacol 9: 312, 2018.

47. Zhai KF, Duan H, Luo L, Cao WG, Han FK, Shan LL and Fang XM: Protective effects of paeonol on inflammatory response in IL-1 $\beta$-induced human fibroblast-like synoviocytes and rheumatoid arthritis progression via modulating NF- $\mathrm{BB}$ pathway. Inflammopharmacology, Aug 10, 2017 (Epub ahead of print)

48. Sun N, Wang H and Wang L: Protective effects of ghrelin against oxidative stress, inducible nitric oxide synthase and inflammation in a mouse model of myocardial ischemia/reperfusion injury via the HMGB1 and TLR4/NF- $\mathrm{BB}$ pathway. Mol Med Rep 14: 2764-2770, 2016.
49. Saraste A, Pulkki K, Kallajoki M, Henriksen K, Parvinen M and Voipio-Pulkki LM: Apoptosis in human acute myocardial infarction. Circulation 95: 320-323, 1997.

50. Fliss $\mathrm{H}$ and Gattinger D: Apoptosis in ischemic and reperfused rat myocardium. Circ Res 79: 949-956, 1996.

51. Haunstetter A and Izumo S: Apoptosis: Basic mechanisms and implications for cardiovascular disease. Circ Res 11: 1111-1129, 1998.

52. Guo M, Chen K, Lv Z, Shao Y, Zhang W, Zhao X and Li C: Bcl-2 mediates coelomocytes apoptosis by suppressing cytochrome c release in Vibrio splendidus challenged Apostichopus japonicus. Dev Comp Immunol 103: 103533, 2019.

53. Zhai KF, Duan H, Chen Y, Khan GJ, Cao WG, Gao GZ, Shan LL and Wei ZJ: Apoptosis effects of imperatorin on synoviocytes in rheumatoid arthritis through mitochondrial/caspase-mediated pathways. Food Funct 9: 2070-2079, 2018.

(i) $\Theta$ This work is licensed under a Creative Commons Attribution-NonCommercial-NoDerivatives 4.0 International (CC BY-NC-ND 4.0) License. 